\title{
Chinese alligator genome illustrates molecular adaptations
}

\author{
Cell Research (2013) 23:1254-1255. doi:10.1038/cr.2013.134; published online 24 September 2013
}

In the September issue of Cell Research, Wan et al. analyze the Chinese Alligator genome and identify multiple instances of positivelyselected changes in protein-coding genes and expansions of multi-gene families that appear to explain suites of phenotypes central to the unique habits and physiology of crocodilian reptiles. They demonstrate the effectiveness of comparative systems genomics in integrating information from comparative genomics, molecular evolution, and systems biology to understand the evolutionary dynamics of complex systems.

The molecular changes that underlie the great diversity of form and function across vertebrates are largely unknown. Phenotypic novelty and diversity may be driven by changes in gene regulation, but changes in the sequence and function of protein-coding genes or expansion and contraction of multi-gene families may also contribute. Wan et al. [1] found multiple examples of protein adaptation and gene family expansions that were a key source of phenotypic novelty, supporting the latter view.

With the first full analysis of a crocodilian genome, Wan and colleagues also fill a major gap in our understanding of amniote genome diversity. Amniotes comprise $\sim 25000$ living species, traditionally split into mammals and "reptiles" (including birds). Modern estimates of amniote relationships indicate that mammals and reptiles are sister lineages [2, 3], and Wan et al. estimate that the split occurred $\sim 241$ Mya. About 2/5 of amniotes are nonavian reptiles (turtles, crocodilians, lizards, snakes, and tuataras), but they have been under-sampled compared to birds and mammals. The only previous complete non-avian reptile genomes were the anole lizard [4] and three turtles [2,3]. Ongoing genome projects and partially-analyzed draft genomes include multiple snake species $[5,6]$ and additional crocodilian species [7]. Endemic to China, the Chinese Alligator (Alligator sinensis) is one of only 24 living crocodilian species worldwide, and its genome begins to correct that disparity.

Crocodilians as a group are top predators in aquatic environments, and have evolved numerous specializations to ambush prey from underwater and to remain submersed for long periods of time. To facilitate this, they evolved physiological adaptations to sense prey, shunt blood differentially to the brain while diving, and control metabolic activity during dives. Wan and colleagues therefore focused on analyses of genes involved in these processes, and how positive selection on protein-coding genes and expansion of multi-gene families have together facilitated the evolution of these phenotypes. They provide strong evidence for coordinated adaptation across multiple functionallyrelated proteins (Figure 1).

The most obvious adaptive pressure likely to arise from needing to hold one's breath during long dives is on oxygen transport systems, and for such genes the Chinese Alligator does not disappoint. Gene duplications led to crocodilian-specific hemoglobins that appear designed for diving, particularly in their switch to bicarbonate preference, which increases $\mathrm{O}_{2}$ release. $\mathrm{O}_{2}$ unloading was also apparently enhanced by positive selection in the gene for anion exchanger 1 . At the metabolic level (Figure 1), there was positive selection on genes associated with oxidative phosphorylation, and also on genes involved in glycan, fatty acid, and terpenoid metabolism. These positively selected changes are thought to further enhance the ability of crocodilians to tolerate the metabolic demands associated with diving for long periods. Selection in other genes may have played roles in facilitating a slower heart rate and fluctuations in cardiac demands associated with diving. Lastly, multiple genes involved in nitrogenous waste excretion experienced positive selection, apparently to accommodate $\mathrm{CO}_{2}$ excretion as ammonium bicarbonate in urine to reduce requirements for lung ventilation while diving.

Crocodilians also need to heal from wounds in bacteria-rich environments. This is reflected in rapid evolution of genes associated with the immune response, along with expansion of alligator-specific immune gene families and antimicrobial peptides (Figure 1). Predators must also hunt, and the Chinese Alligator shows clear signs that its senses have adapted towards success in this pursuit. There were alligatorspecific gene expansions in olfactory receptors along with rapid evolution, a finding that explains the alligator's keen sense of smell. In addition, there was positive selection on synaptic genes, neurological system processes and nerve-related cell components, and on auditory and ocular genes (Figure 1). Opsin, for example, is important in sensing light and dark, while otopetrin is involved in development of the sound- 


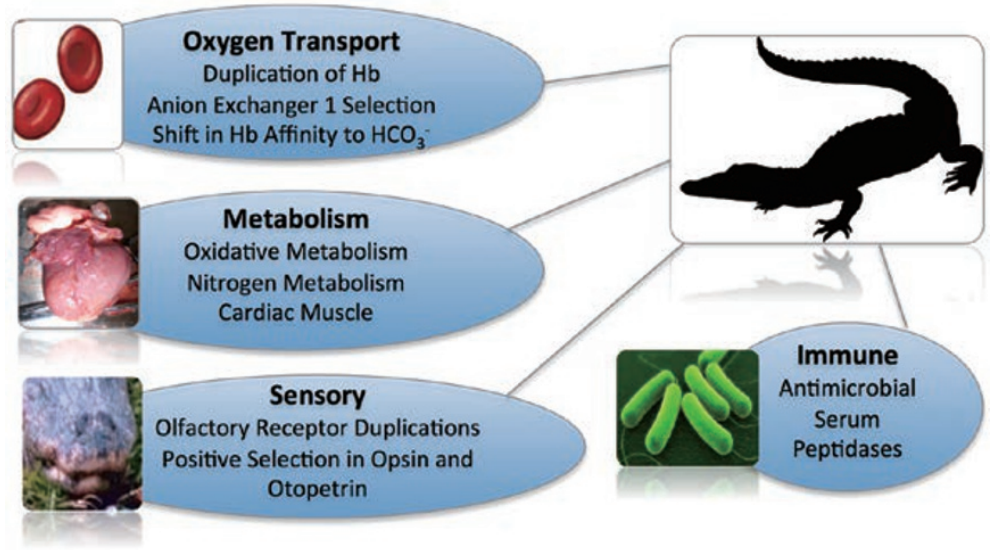

Figure 1 Major systems shown to have adaptively changed via protein evolution and gene family expansion in the lineage, leading to the Chinese Alligator. $\mathrm{Hb}$, hemoglobin; OR, olfactory receptor.

detecting otoliths, and both experienced positive selection in crocodilians.

Ironically, despite showing that many genes underwent accelerated evolution, Wan and colleagues also show that most of the crocodilian genome evolved quite slowly. Transposable elements and segmental duplications (SDs) in the Chinese Alligator genome appear ancient compared to other reptiles and birds. This implies less sequence turnover and fragmentation, and suggests that crocodilians may be invaluable in reconstructing ancestral amniote genomes. Additionally, a correlation between LINE retrotransposons and SD distributions among reptilian genomes implies that LINEs may play an important role in generating SDs.

Adaptive changes in core metabolic processes are starting to appear as a recurring theme in vertebrate evolution. Snakes and other lizards underwent major evolutionary redesign of core oxidative metabolism genes, presumably driven by selection for low resting metabolic rates and the ability to fluctuate widely among metabolic rates $[8,9]$. Similar but less extreme genetic shifts also occurred in cetaceans [10] and primates [11]. Such shifts may therefore be required to fine-tune the fit of metabolic processes to the specialized physiological requirements of different vertebrates.

The increasing availability of diverse amniote genomes is exciting because they contribute substantially to the understanding of the genetic basis of how changes in physiology were accomplished. Yet we still need to more accurately assess the timing of major shifts in genome composition and molecular adaptations on the amniote tree. For example, we do not know whether molecular adaptations identified occurred in the ancestor of all living crocodilians, or only on the Chinese Alligator lineage. Furthermore, there are currently too few reptile genomes available to accurately assess the degree to which rates and patterns of change vary across lineages. Nonetheless, the contributions of Wan and colleagues are substantial, fill major gaps in our understanding, and provide inspiring evidence of what can be learned about the molecular underpinnings that have driven amniote physiological and phenotypic diversity.

\section{Todd A Castoe ${ }^{1}$, David D Pollock ${ }^{2}$}

${ }^{1}$ Department of Biology, University of Texas Arlington, Arlington, TX 76010, USA; ${ }^{2}$ Department of Biochemistry and Molecular Genetics, University of Colorado School of Medicine, Aurora, CO 80045, USA

Correspondence: David D Pollock

E-mail: David.Pollock@UCDenver.edu

\section{References}

1 Wan QH, Pan SK, Hu L, et al. Cell Res 2013; 23:1091-1105.

2 Shaffer HB, Minx P, Warren DE, et al. Genome Biol 2013; 14:R28.

3 Wang Z, Pascual-Anaya J, Zadissa A, et al. Nat Genet 2013; 45:701-706.

4 Alfoldi J, Di Palma F, Grabherr M, et al. Nature 2011; 477:587-591.

5 Castoe TA, Bronikowski AM, Brodie ED 3rd, et al. Stand Genomic Sci 2011; 4:257270.

6 Castoe TA, de Koning JA, Hall KT, et al. Genome Biol 2011; 12:406.

7 St John JA, Braun EL, Isberg SR, et al. Genome Biol 2012; 13:415.

8 Castoe TA, de Koning AP, Kim HM, et al. Proc Natl Acad Sci USA 2009; 106:89868991.

9 Castoe TA, Jiang ZJ, Gu W, et al. PLoS One 2008; 3:e2201.

10 McClellan DA, Palfreyman EJ, Smith MJ, et al. Mol Biol Evol 2005; 22:437-455.

11 Grossman LI, Wildman DE, Schmidt TR, et al. Trends Genet 2004; 20:578-585. 